\title{
Revealing perovskite formation kinetics during chemical vapour deposition
}

Received 00th January 20xx, Accepted 00th January 20xx DOI: $10.1039 / \times 0 \times x 00000 x$
Thierry A. Moser ${ }^{a}$, Kerem Artuk ${ }^{a}$, Yan Jiang*a, Thomas Feurer ${ }^{a}$, Evgeniia Gilshtein ${ }^{a}$, Ayodhya N. Tiwari ${ }^{\mathrm{a}}$ and Fan Fu*a
Amongst a number of deposition methods for perovskite layeßs 1 vapour based ones are promising for large area industrỉ $\mathbb{P}$ production of solar cells. Different variants of such methods $a B B$ high efficiencies have been reported recently, but there remain $3 A$ lack of understanding on the formation process of perovskite laye 35 with 2-step vapour deposition. Here, we present a new react36 design for controlled investigation of the reaction kinetics $B B$ conversion of evaporated metal halide precursor layer (such as 38 mixture of lead iodide and cesium bromide) into perovskite lay 3 by exposure to an organo-halide (such as formamidinium iodidł ( vapour under stable isobaric-isothermal conditions. With this nethl concept of gas flow reversal in tubular reactor, we overcome 2 inherent problem of the lack of control over the precise start aAB end of the conversion process. We investigated the formatiant reaction of mixed cation $\left(\mathrm{Cs}_{0.04} \mathrm{FA}_{0.96}\right) \mathrm{Pbl}_{3}$ perovskite in well-defin $A \dot{\Phi}$ intermediate states to elucidate the influence of processiA $\$$ conditions on the kinetics of perovskite and other phated formations. High conversion rate of up to $60 \mathrm{~nm} / \mathrm{min}$ is achievA\$ with a well-controlled abrupt start and end of the vapor suppA99 Using our deposition method, a semitransparent solar cell with $5 \$$ power conversion efficiency (maximum power tracking) of $9.6 \% \mathrm{bn}$ a designated area of $0.27 \mathrm{~cm}^{2}$ is achieved in the initial phase 52 development where the charge extracting layers and interfaces yet to be optimised.

The past decade has witnessed the unprecedented advances 5,8 power conversion efficiency (PCE) of thin film solar cells based on metal halide perovskites $\left(A B X_{3}, A=C s\right.$, methylammoniư 9 (MA), formamidinium (FA); $B=P b, S n ; X=C l, B r,{ }^{1,2}$ ), with 61 certified efficiency at $25.2 \%$ on $0.1 \mathrm{~cm}^{2} .3,4$ An industriaff?

a. Laboratory for Thin Films and Photovoltaics, Empa - Swiss Federal Laboratories ; for Materials Science and Technology, Ueberlandstrasse 129, Duebendorf 8600, ; Switzerland.E-mail: fan.fu@empa.ch; yan.jiang@empa.ch promising approach for the deposition of perovskite thin films is the chemical vapour deposition $(C V D)^{5,6}$. Although in literature various technical implementations and also naming (e.g. H-CVD: hybrid chemical vapour deposition ${ }^{7}$, VASP: vapour assisted solution processing ${ }^{8}$, ITCVD: in-situ tubular chemical vapour deposition ${ }^{9}$, LP-CVD: Low pressure $C \mathrm{CV}^{10}$ ) of this method can be found, the underlying principle is the same: An organo-halide material (e.g. FAl) is sublimed and its vapour is transported to the substrate, where it reacts with a predeposited metal halide precursor layer (e.g. a CsBr/Pbl 2 ).

While this precursor layers can be deposited from solution ${ }^{11,12}$, they can also be thermally evaporated, which maintains the advantage of high-uniformity and scalability. Tong et al. converted a sequentially evaporated $\mathrm{CsBr} / \mathrm{Pbl}_{2}$ stack with FAI for 100 minutes at $140^{\circ} \mathrm{C}$ to realize a compositionally graded absorber and achieved an opaque device with a PCE of $18.2 \%$ $\left(0.09 \mathrm{~cm}^{2}\right)^{13}$. Qiu et al. also employed an all-vapour approach by converting a co-evaporated $\mathrm{CsBr} / \mathrm{Pbl}_{2}$ precursor with $\mathrm{FAI}$ vapour. They realized an opaque mini-module with a designated area of $91.8 \mathrm{~cm}^{2}$ and $9.34 \%$ PCE. This corresponds to a loss of only $1.3 \% /$ decade of scaled area when comparing to their champion efficiency of $13.3 \%$ on $0.09 \mathrm{~cm}^{2} .^{14}$

Despite the advances in power conversion efficiency, perovskite formation kinetics during the CVD process has remained elusive. The CVD process is usually carried out in a reaction chamber that can be heated and evacuated to provide suitable sublimation and reaction conditions. Under such conditions, both the sublimation and the transport of the organic species already starts and accelerates during the heat-up period and also doesn't immediately stop during the cool-down period. This means that the conversion reaction is not happening under constant conditions but continuous changes in temperature and vapour pressure of the organic species are occurring. But in order to study the perovskite formation kinetics during the CVD process, stable conditions and a well-defined reaction time window are required.

Electronic Supplementary Information (ESI) available: [details of any supplementary information available should be included here]. See DOI: 10.1039/x0xx00000x

This document is the accepted manuscript version of the following article: Moser, T., Artuk, K., Jiang, Y., Feurer, T., Gilshtein, E., Tiwari, A. N., \& Fu, F. (2020). Revealing the perovskite formation kinetics during chemical vapour deposition. Journal of Materials Chemistry A. https://doi.org/10.1039/D0TA04501B 
a

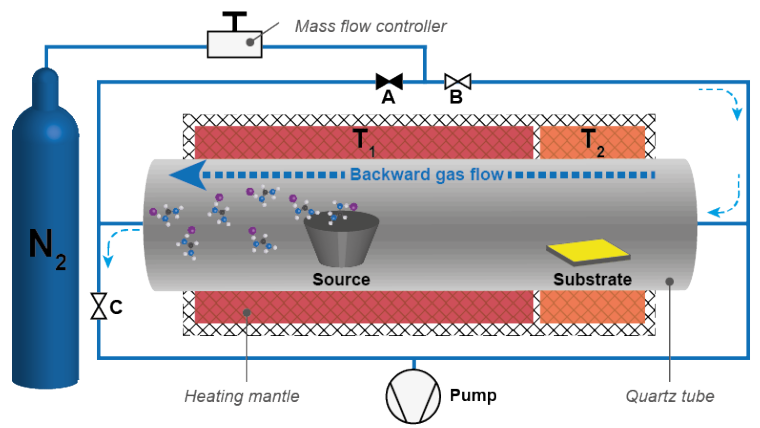

b

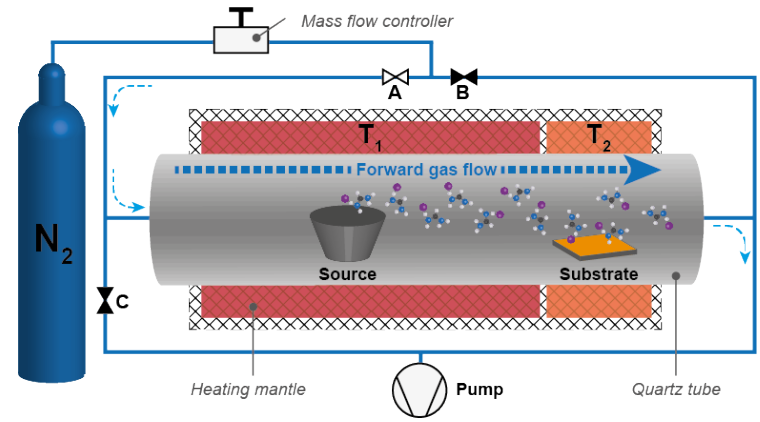

C

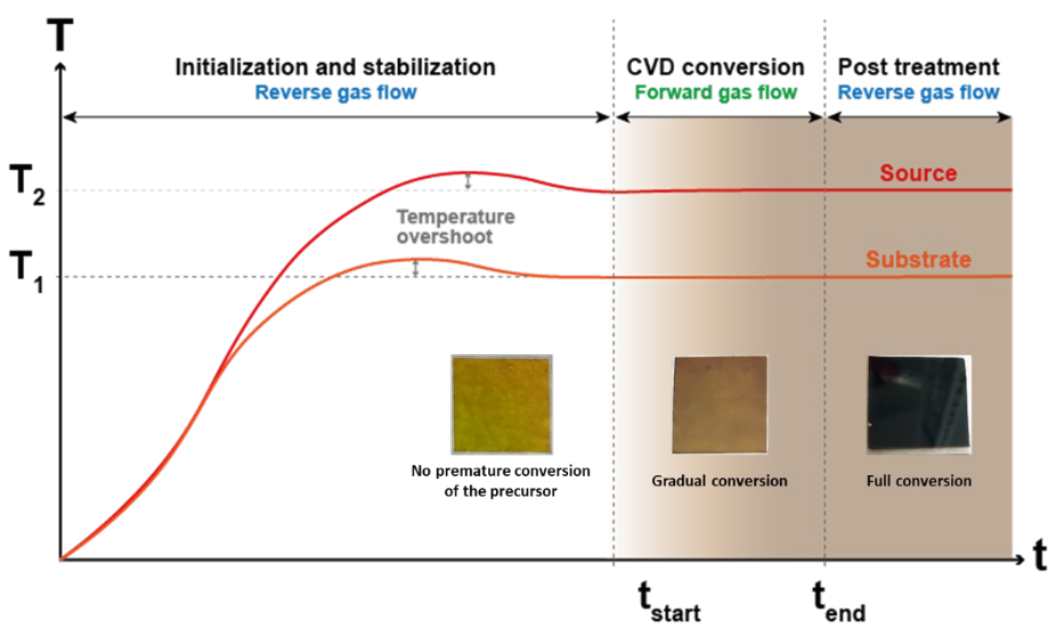

Figure 1: Controlled isothermal-isobaric CVD process. a) and b) Schematic of the CVD reactor, allowing quick reversal of carrier gas flow. Depending on the flow direction, the premature reaction process is prevented before attaining the stabilized thermal conditions. c) Time-temperature-dependent reaction graph with control of carrier gas flow direction: Reverse gas flow allows to stabilize the temperature zones without occurrence of premature conversion reaction. Changing the gas flow direction allows to start and stop the conversion reaction. Additionally, the sample can be kept in the reactor for a post conversion annealing treatment if needed.

In this work, we present the deposition of a mixed catiob $\left(\mathrm{Cs}_{0.04} \mathrm{FA}_{0.96}\right) \mathrm{Pbl}_{3}$ perovskite via a hybrid chemical vapog 1 deposition process where we are able to achieve the requirgd control by decoupling the heating and reaction step. Through the systematic study of the perovskite film formation kinetig4 we find that the perovskite formation reaction is either mas 95 transport or diffusion-limited, depending on the temperature $9 f$ the FAl sublimation zone. Furthermore we find that tge conversion reaction kinetics are not only controlled by the FAd sublimation temperature but also the substrate temperaturg9 Understanding the kinetics allows us to deposit high-qualito perovskite film with a growth rate of up to $60 \mathrm{~nm} / \mathrm{mil}_{1}$ Additionally, we observe the formation of additional phape 2 besides the desired perovskite under certain processibs conditions. These side reactions have to be avoided 1004 therefore are important to consider for the CVD deposition 105 phase pure perovskite absorbers. As proof-of-concept, the 1606 process was used to fabricate a semi-transparent PSC withp@y any post-treatment of the absorber or optimization of 108 contact layer. The final device exhibits a power conversig? efficiency of $10.6 \%$.

\section{Isothermal-isobaric CVD process}

In the works published so far on the subject of perovskite absorbers deposited via CVD we encounter systems where the organic vapour is allowed to come into contact during the complete process. This means that the conversion already starts (resp. continues) during the heat-up (resp. cool-down) of the system. Therefore the conversion is not occurring under constant conditions (i.e. substrate/source temperature, pressure, concentration of the organic vapour).

To achieve better control over the CVD process we modified the classical concept of a tubular reactor. Our set-up allows for the selective injection of the carrier gas from either side of the reaction chamber. Thus, a gas flow in both directions is possible - an approach that has yet not been employed in perovskite research. With our set-up, we can at first have a carrier gas flow in the reverse direction (i.e., going from the substrate side towards the source side), as depicted in Figure 1a. Under this condition, we ramp the two heating zones to their distinct target temperatures with the carrier gas preventing premature contact between the organic vapour and the precursor. Once pressure and temperatures stabilized, the flow direction of the carrier gas is reversed, enabling the vapour and precursor layer to get into contact (see Figure 1b). Reversing the flow direction 113 a second time allows stopping the conversion reaction. 
Table 1: Conditions studied for their effects on reaction kinetics and phase evolution.

\begin{tabular}{|c|c|c|}
\hline Condition & Substrate temp. $\mathrm{T}_{2}\left[{ }^{\circ} \mathrm{C}\right]$ & Source temp. $\mathrm{T}_{1}\left[{ }^{\circ} \mathrm{C}\right]$ \\
\hline A & 160 & 190 \\
\hline B & 180 & 190 \\
\hline C & 160 & 210 \\
\hline D & 180 & 210 \\
\hline
\end{tabular}

114 In short, our CVD setup allows the conversion reaction to happen in isothermal and isobaric conditions for a well-defined time window. While this is not expected to directly improve the quality of the final perovskite layer, this unlocks the possibility to conduct time-resolved mechanism studies on the effect of temperature and pressure conditions during the CVD proces $\$ 57$ For our study we employed a co-evaporated $\mathrm{CsBr} / \mathrm{PbI}_{2}$ la1 188 with an atomic percentage of $4 \% \mathrm{CsBr}$ as confirmed by $\mathrm{X}-\mathrm{r} 59$ photoelectron spectroscopy (XPS; Figure S1) and convert it vifio our CVD set-up with FAl vapour. This composition was cho\$1 as cesium and formamidinium based perovskites have sho16 2 high stability and power conversion efficiencies. ${ }^{15-17}$ Albeit the precursor contains bromine, XPS did not show 164 presence in the final perovskite (Figure S2-S3) as it is repla165 by iodine by ion exchange during the CVD process.

\section{Kinetics of the conversion reaction}

To investigate the reaction kinetics of the CVD process, 169 estimated the conversion fraction $\Gamma(t)$ of the precursor laty after different reaction times $t$ as this gives a measure for the progress of the reaction. Following the approach 172 Ummadisingu et al. ${ }^{18}$ the conversion fraction was defined as 173

$$
\Gamma(t)=\frac{A(t)}{A_{f c}} * 100 \%
$$

where $A(t)$ is the absorbance of the mixed perovskite layer aftes reaction time $t$ and $A_{f c}$ is the absorbance of a fully converfed layer $(\sim 500 \mathrm{~nm})$. This conversion fraction was calculated frppo UV-Vis spectroscopy data using the Lambert-Be\&5 relationship $^{19}$. Details on the optical model applied, 182 underlying calculations are given in the supplementary information (Figure S4-S6).

Figure 2 shows the conversion fraction as a function of reaction time for four different temperature conditions, specified 186 Table 1. For all the runs precursor thickness and composition 187 well as pressure and mass flow of the carrier gas were kept 188 same.

The fastest kinetics is observed for condition C (160-210), wherfo a full, homogeneous conversion of a $250 \mathrm{~nm} \mathrm{CsBr} / \mathrm{P}_{12}$ precursor layer is achieved in only 8 minutes. In literature, the required time of the CVD conversion process.jं often between one and three hours 9,12,20. This is due to slow thermal response of conventional tube furnaces and 195 low heating rates that need to be employed to avoid therpal overshoots. Hoerantner et al. on the other hand, reported 197

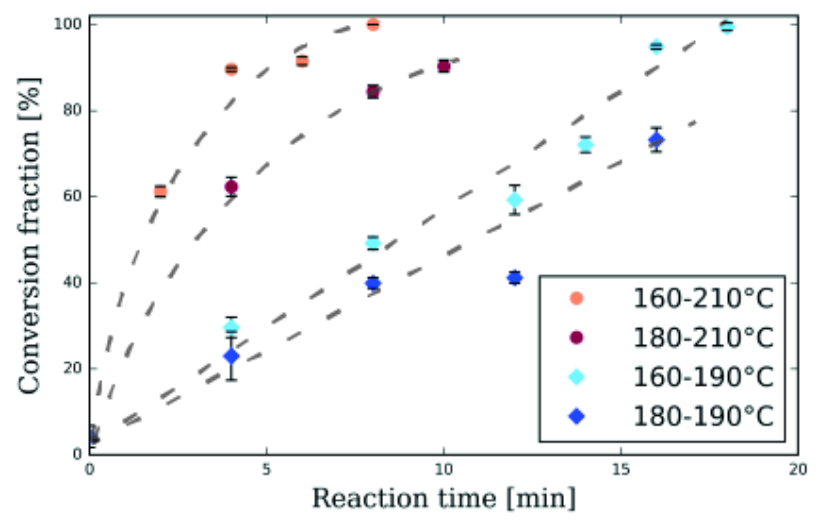

Figure 2: Reaction kinetics of perovskite formation at different conditions shown by the change of the conversion fraction under different conditions with time. The dashed lines serve as guide for the eye.

high-speed vapour deposition technique that only requires a three-minute-long step for the conversion of a $200 \mathrm{~nm}$ thick $\mathrm{Pbl}_{2}$ precursor layer into $\mathrm{MAPbl}_{3}$ perovskite ${ }^{21}$. This already demonstrated that the actual conversion reaction can be achieved at shorter times. In an industrial in-line system, where the substrate would be continuously fed into the reactor and an initialization step is not needed, the actual required conversion time is the key parameter that determines the maximally achievable throughput. For the design of the CVD equipment such knowledge of the reaction kinetics is essential.

One can see that a higher source temperature $T_{1}$ strongly increases the conversion rate. This shows that the speed of conversion can be controlled by the sublimation rate of the FAl. Possibly, the rate of conversion could be even further accelerated by increasing the source temperature. But such high kinetics might impede the controllability and reproducibility of the process unless sophisticated temperature and gas flow controls are used.

Comparing the slow and fast (i.e., low resp. high source temperature $T_{1}$ ) conversion regime, we observe two different behaviour. When working with a low source temperature (i.e., condition A (160-190) and B (180-190) ), the conversion rate is constant and not affected by the increasing thickness of the perovskite layer. In the case of high source temperature (i.e., condition C (160-210) and D (180-210)) we observe a decrease in the conversion rate with time. This indicates that at a low source temperature, the reaction kinetics are governed by the mass-transport of the FAl vapour. The decrease in conversion rate at a high substrate temperature implies that the solid-state diffusion becomes the dominant factor.

While the influence of the source zone temperature $T_{1}$ on the reaction kinetics was anticipated, the influence of the substrate zone temperature $T_{2}$ shows the reverse effect: For both slow and fast conversion regime, the reaction speed was lowered when a higher substrate zone temperature $T_{2}$ was chosen. $A$ higher substrate temperature $T_{2}$ usually is expected to provide more thermodynamic driving force for the reaction between the reactants and also facilitate the interdiffusion, so one could expect to observe an acceleration of the reaction. Nevertheless, it is important to bear in mind that the substrate temperature both affects the condensation dynamics of the FAl vapour and 

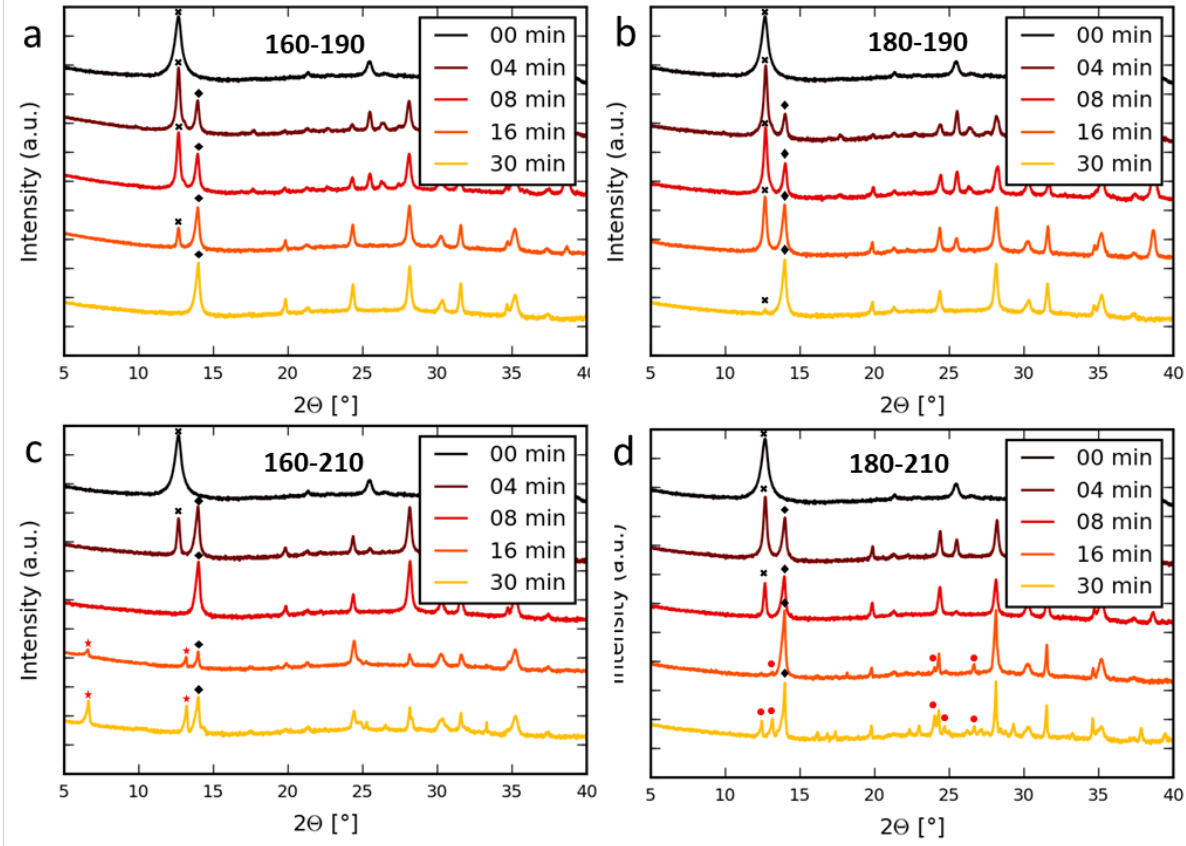

e

$\mathrm{T}_{1}: 160^{\circ} \mathrm{C} / \mathrm{T}_{2}: 190^{\circ} \mathrm{C}$
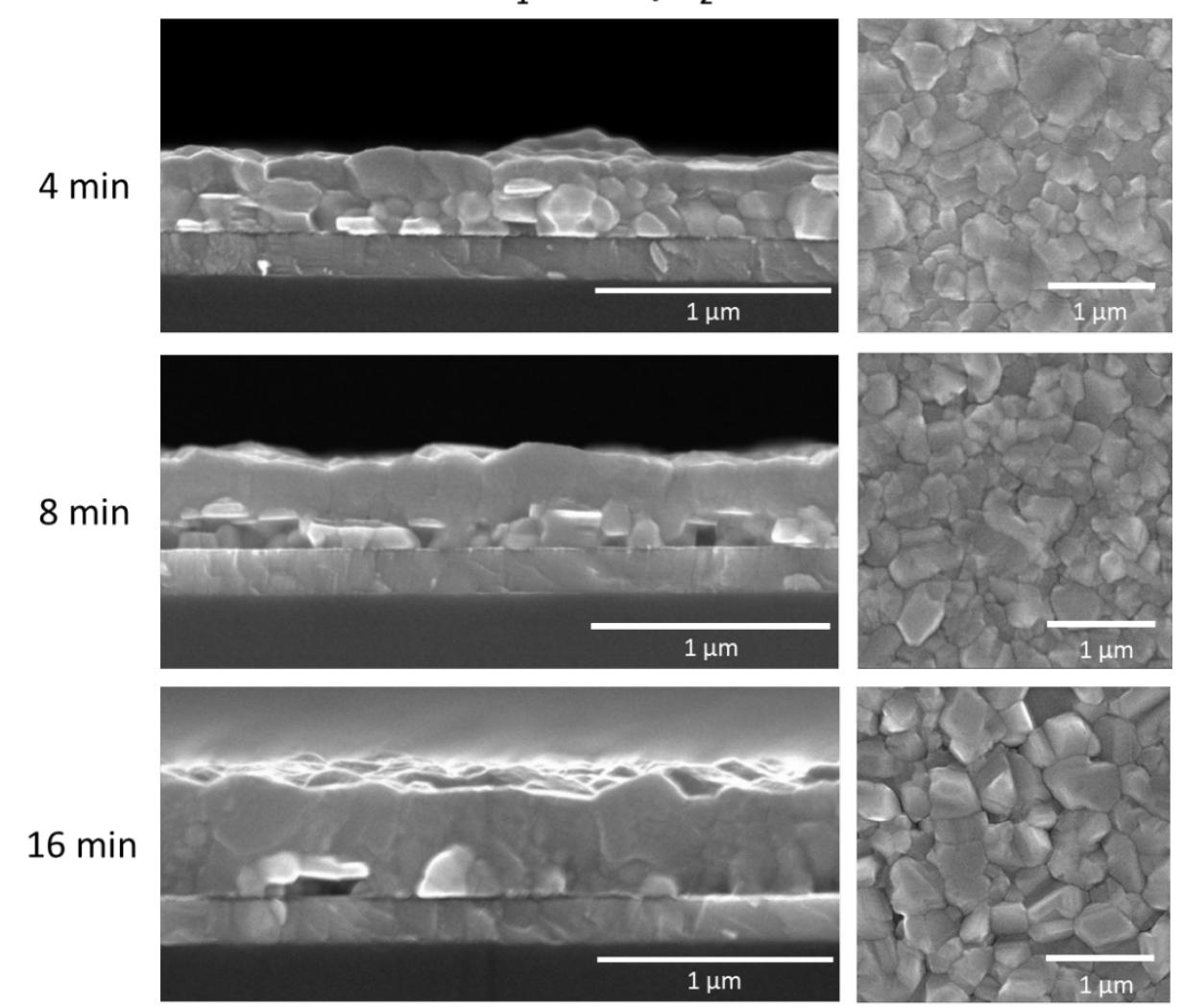

Figure 3: Phase evolution during CVD conversion of perovskite for different conditions of reaction time and temperatures (in ${ }^{\circ} \mathrm{C}$ ) of substrate and evaporation source. a) $160-190$, b) $180-190$, c) $160-210$, d) $180-210$. During the perovskite formation, the precursor peak (indicated by $\mathbf{x}$ ) at $12.7^{\circ}$ is continuously fading and the perovskite phase peak (indicated by $\bullet$ ) gains intensity. In c) and d) the formation of additional 'overconversion' phases (indicated by $\bullet$ and $\star$ ) is observed once the precursor is converted completely. e) SEM crosssection and top-views of layers after $4 \mathrm{~min}, 8 \mathrm{~min}$ and 16 min reaction time with the temperature zone at $160^{\circ} \mathrm{C}$ and the source zone at $190^{\circ} \mathrm{C}$

198 the decomposition reaction of the perovskite. A loखथ2 199 temperature $T_{2}$ will result in higher rate of condensation on $\mathbb{Z}$ B 3 200 substrate surface (i.e., a higher supply of FAl to the convers 204 201 reaction) and a slower decomposition reaction. These 2005 effects lead to the accelerated kinetics of the conversion reaction at a lower temperature $\mathrm{T}_{2}$. 


\section{Phase evolution of conversion reaction} peak intensities.
To probe the phase evolution during the CVD process, we performed X-ray diffraction measurements on the thin films converted at the four different conditions for various times. These phase evolutions are depicted in Figure 3. The two main peaks at $12.7^{\circ}$ and $14.0^{\circ}$ correspond to the $\mathrm{Pbl}_{2}(110)$ and the (110) perovskite crystalline planes, respectively. They reflect the conversion process under different conditions: For the fast conversion regime (i.e., $\mathrm{T}_{1}=210^{\circ} \mathrm{C}$ ) the peak of the precursor phase vanishes and the perovskite peak rises very quickly with time while these effects are more gradual for the slow conversion regime (i.e., $\mathrm{T}_{1}=190^{\circ} \mathrm{C}$ ). Again, we observe that a higher substrate temperature $T_{2}$ causes a slower change of the

Under conditions C (160-210) and D (180-210) additional phases are evolving after the precursor has transformed into perovskite. Under condition C (160-210), two new peaks skaf? to show up at $6.6^{\circ}$ and $13.2^{\circ}$ for a conversion time of 16 minutete and more. Under condition $D(180-210)$ new peaks are prese 244 at $12.5,24.0^{\circ}, 24.7^{\circ}$ and $26.7^{\circ}$ when the CVD process is run $28^{5}$ 16 minutes or more. Formation of these new phases 26,6 accompanied by strong changes in optical properties. Initiafi7 the transparent yellow precursor layers turn to a da brown/black appearance indicating the conversion 269 perovskite. Subsequently the appearance would change agatip depending on the conditions of the process. Under conditioff $(160-210)$ the layers turned to an inhomogeneous yellä 2 orange hue, while for condition $D(180-210)$ the layers shova $2{ }^{3}$ a grey, milky colour and macroscopic cracks. The differeht appearances can be seen in Figure $5 f$. We would like to pơih out that the apparent inhomogeneity of the precursor layer $277^{\circ} \bar{p}$ the layers converted at $170-180^{\circ} \mathrm{C}, 160-190^{\circ} \mathrm{C}$ and $220-200^{\circ} \mathrm{Z} 7 \mathrm{7}$ those photographs stems from the background paper substraze and is not due to the layers themselves. A possible explanation for the emergence of these additiorfip peaks could be the condensation and crystallization of ptipt source material (i.e., formamidinium iodide) on the sample. 7 IF $^{2}$ XRD pattern taken from pure formamidinium iodide powder 89 displayed in Figure $\mathbf{S 7}$ and no overlap of the diffraction pe $\mathbf{2 8} 8$ with the ones observed in our CVD samples could be found. 2185 therefore assume that these additional phases stem from 286 reaction between the already formed perovskite phase and 288 formamidinium vapour. This conclusion is in line with w28 18 conducted by Leyden et al. where during CVD-deposition289 $\mathrm{FAPbl}_{3}$ foreign phases together with variations of the opt2090 appearance were observed, which they attributed to a FAIphase $^{20}$. We term such layers with additional phases as 'opgz converted'. To study the morphological evolution of 29 perovskite formation, we examined the different stages vigh secondary-electron microscopy (SEM). Figure 3e shows the to95 view and cross-sectional SEM micrographs of the layzeg6 processed under condition A (160-190) for 4, 8 and 16 minu29 7 The series of cross sections shows that the perovskite ph 28 forms a continuous layer over the thinning precursor layer 2 continues growing until only single grains of precursor are BefO at the perovskite-substrate interface. In general, the conversion

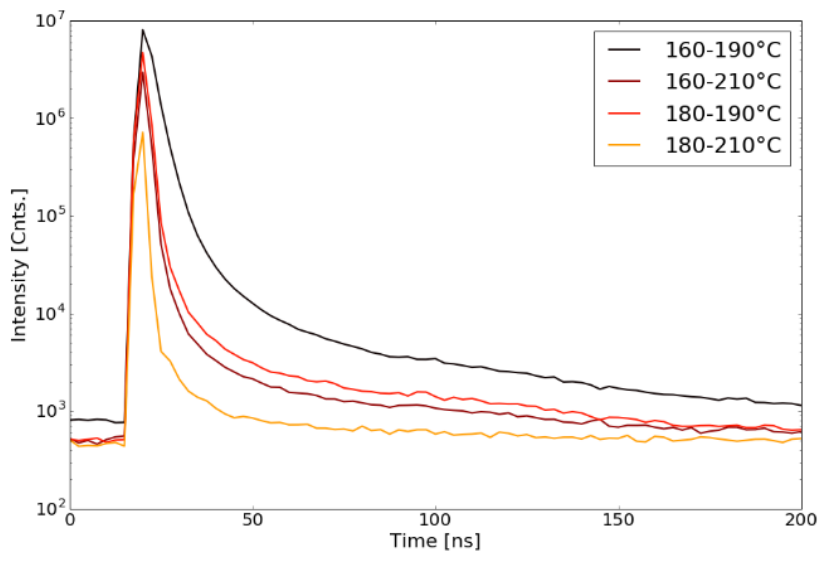

Figure 4: TRPL measurements of perovskite films on fused silica grown under different CVD conditions.

occurs uniformly and creates a flat and pin-hole free perovskite layer. We assessed the grain size distribution for the four different conditions from top-view SEM micrographs (see Figure S11), which show that that a faster conversion reaction favours the formation of smaller grains. This observation can be explained by the common theory of crystallization: A faster conversion reaction is expected to create a larger number of nucleation sites where the perovskite crystals can grow, thus creating many small crystallites. Additionally, the reaction time determines the Ostwald ripening. For a slow conversion, the opposite effects, i.e. a lower number of nucleation sites and a higher degree of Ostwald ripening, are expected. The final grains of the perovskite show diameters of a few hundred nanometers. The corresponding micrographs are shown in Figure 59-S11. As no that the final morphology is likely to be mainly determined by other parameters (such as the morphology of the metal halide precursor and the pressure during (VD) than the temperature respectively the growth rate.

\section{Time-resolved photoluminescence results}

Figure 4shows the result of time-resolved photoluminescence (TRPL) measurements conducted on perovskite layers grown on fused silica under the four different conditions A-D. A higher luminescence intensity and a longer carrier lifetime was observed for a slow conversion and a low substrate temperature during the conversion.

The TRPL decay shows a very fast quenching of the photoluminescence. This indicates that the recombination of the charge carriers is strongly dominated by non-radiative recombination. From a bi-exponential fitting we estimated a radiative lifetime of $55 \mathrm{~ns}$ for CVD condition $A\left(160-190^{\circ} \mathrm{C}\right)$. For the conditions $B, C$ and $D$ no acceptable fitting was possible. Such low lifetimes are quite typical and are still one of the major challenges for vacuum-deposited absorbers ${ }^{12,21-23}$. Perovskite bulk is commonly assumed to be highly defect tolerant and mainly the surface defects are thought to be responsible for non-radiative recombination of charge charriers ${ }^{24,25}$. We assume that our vapour phase deposition of perovskite favours 
301 the formation of surface defects, which explains the low car3i5 7

\section{lifetimes of the pristine absorbers.}

\section{Isochronal process map}

To gain further insight into the CVD process and the formation of the 'overconverted' phases, we processed perovskite films 36 a fixed conversion time of 30 minutes and source temperaturies $\mathrm{T}_{1}$ ranging from $180^{\circ}$ to $220^{\circ} \mathrm{C}$ and substrate temperature $5_{2} \mathrm{~S}_{2}$ ranging from $150^{\circ}$ to $220^{\circ} \mathrm{C}$. The results of the $\times 60^{5}$ measurements of the fabricated samples are visualized 36 Figure 5. The intensity maps in Figure $\mathbf{5 a}$ and $\mathbf{b}$ show the $(1987$ perovskite phase reflection at $14^{\circ}$ and the (110) $\mathrm{Pbl}_{2}$ precurs 368 phase reflection at $12.6^{\circ}$, respectively. Additionally, 369 reflections at $6.6^{\circ}$ and at $24^{\circ}$ which stem from the 'overconverted' phase were mapped in Figure $5 \mathbf{c}$ and $\mathbf{d}$. One can see that the perovskite phase is present after processing at nearly each condition except for the extreme caje of a substrate temperature of $220^{\circ} \mathrm{C}$. Meanwhile the precursor is only present for the case of the lowest source temperature (i.e., $\mathrm{T}_{2}=180^{\circ} \mathrm{C}$ ) or a substrate temperature $\mathrm{T}_{2}$ equal or above $200^{\circ} \mathrm{C}$. For the two 'over-converted' phases, we find 377 different regimes where they start to appear. On the one hand 3 the peak at $6.6^{\circ}$ is observed for low substrate temperatures 37.9 $\mathrm{T}_{2}<170^{\circ} \mathrm{C}$ ) together with elevated source temperatures (i.e. $>190^{\circ} \mathrm{C}$ ). On the other hand the peak at $24.0^{\circ}$ is observed +81 medium substrate temperature (i.e., $170^{\circ} \mathrm{C}<\mathrm{T}_{2}<210^{\circ} \mathrm{C} 38$ in combination with high source temperatures (i.e., $\mathrm{T}_{1}>200^{\circ} \mathrm{C} 83$ Based on the XRD maps in Figure 5a-d, we constructed 384 isochronal processing map shown in Figure 5e. The mas visualizes the outcome of the CVD process of 30 minutes, depending on the set temperature of the source zone and $318 \nexists$ substrate zone. For a source temperature $T_{1}$ below $190^{\circ} \mathrm{C}$ we define an are 389 'insufficient FAl sublimation'. In this region, the sublimation $\mathbf{3} 99$ of the source material (i.e., formamidinium iodide) is very 1301 In consequence, the sample is only weakly converted, eß02 after the 30 minutes of processing time. Clearly, this set 393 lower boundary for a useful processing window in which $\mathrm{B} B 4$ desired perovskite absorber can be fabricated. AnotBis 5 boundary is imposed by the thermal stability of the perovsk3 26 For substrate zone temperatures above $200^{\circ} \mathrm{C}$ there is 390 perovskite but only precursor present after the CVD proc 3888 This region is named 'decomposition' as the perovskite 399 formed at all - would not withstand the combination of reduAed pressure and elevated temperature and decompose.

401 The intriguing aspect of this processing map are the 402 different 'overconversion regions' in which additionaAO3 unwanted - phases are formed. This does not only imp@BA additional boundaries to the processing window but it pro 45 that the perovskite phase is not the only product that canADE formed from cesium bromide, lead iodide and formamidinitor iodide. It is important to note that the 'overconverted' phases 400 formed via the perovskite phase, after extended reaction tirfim and at high FAl vapour concentrations. This means that Ald thicker precursor layers can be converted without the formation of the overconverted phase if the appropriate CVD conditions are chosen. We also do not observe a single-phase layer but rather a blend between perovskite and 'overconverted' phase. These observations indicate that we are either dealing with a thermodynamic equilibrium or that the formation of the 'overconverted' phases is kinetically hindered. We see that at the same source temperature (e.g. $200^{\circ} \mathrm{C}$ ) the formation of the 'overconverted' phase can be prevented by using a higher substrate zone temperature. This supports the assumption of a thermodynamic equilibrium. The higher substrate temperature decreases the condensation of the FAI vapour, which reduces its chemical potential in the reaction. If there were a kinetic hindrance, we would expect that the 'overconverted' phase is formed faster at a higher temperature. It is essential to understand under which conditions this 'overconversion' reaction occurs for the sake of achieving high quality perovskite absorbers. We have already observed that the 'overconversion' strongly changes the optical properties of the fabricated layer. Clearly this deteriorates the performance of the layer when used as an absorber in a photovoltaic device. In the work of Leyden et al. they observed similar 'overconversion' behaviour during the CVD deposition of $\mathrm{FAPbl}_{3}$ but did not further investigate under which CVD conditions it occurred $^{20}$. They also showed that the phase formation was reversible, which supports the notion of a thermodynamic equilibrium. Still the final device performance was impaired by the reversal, indicating that it is better to completely avoid the overconversion reaction.

\section{Scalability}

To demonstrate the scalability of the CVD process, we converted a precursor layer deposited on a $5 \times 5 \mathrm{~cm}^{2}$ fused silica substrate and measured the UV-Vis response and XRD patterns in 9 different areas. The XRD shows a single phase perovskite layer in all probed areas without any precursor or overconversion phase present. From the UV-Vis results we see that the perovskite layer is thickest in the middle of the substrate and up to $10 \%$ thinner in the edge/corner regions. This non-uniformity is not stemming from the CVD conversion but rather from the PVD deposition of the precursor. Profilometry measurements show that the precursor template also exhibits a $\sim 10 \%$ lower thickness in the edge/corner regions. These results suggest that we can uniformly convert precursor layers on larger substrates. Still it is important to mention, that although a high source temperature (i.e. $\mathrm{T} 1=210^{\circ} \mathrm{C}$, high FAI vapour concentration) was used, the reaction time had to be extended to 20 minutes to achieve full conversion. This shows that our experimental CVD set-up is susceptible to the precursor area present. Nevertheless, we are convinced that the high growth rate achieved on small substrates would be feasible with CVD in a uniform manner with appropriate reactor design. The corresponding graphs can be found in Figure S12. 


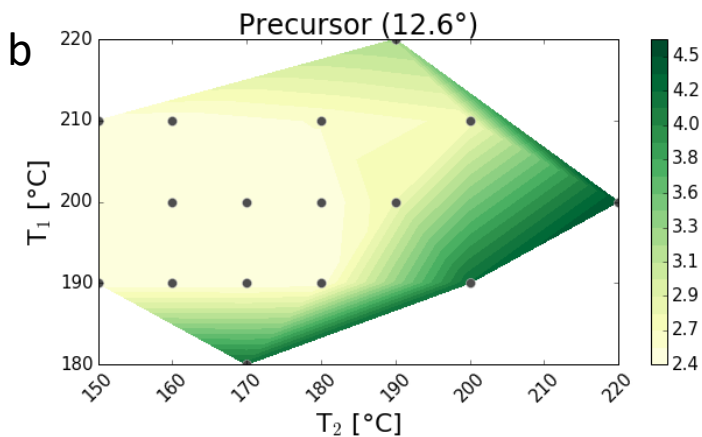

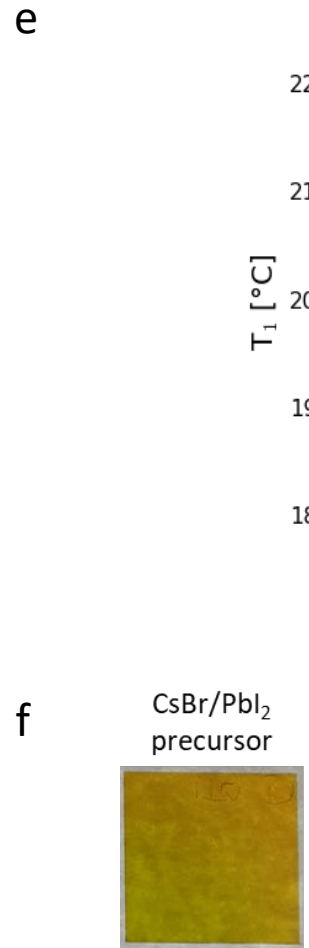

no

conversion

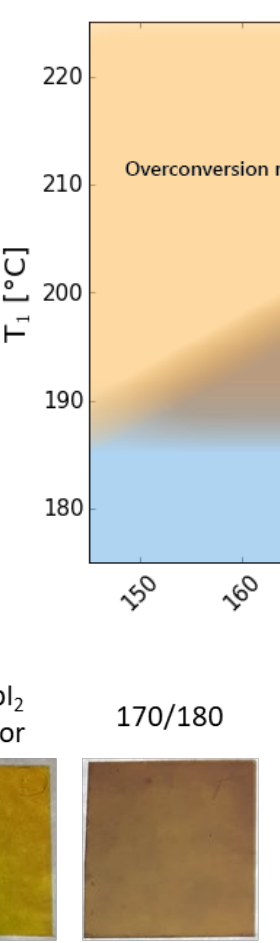

under-

conversion
COMMUNICATION
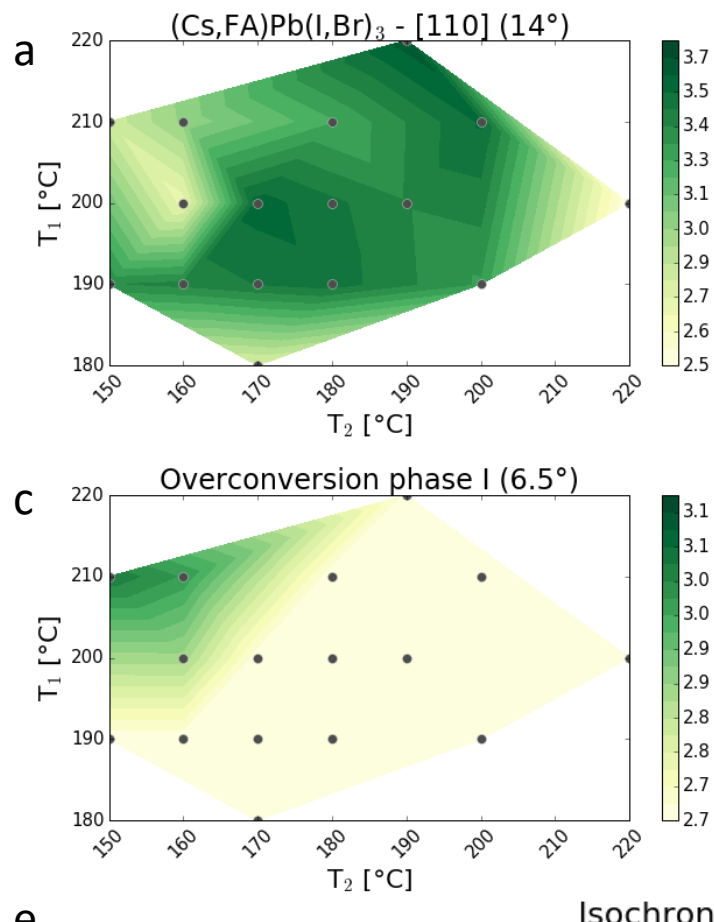

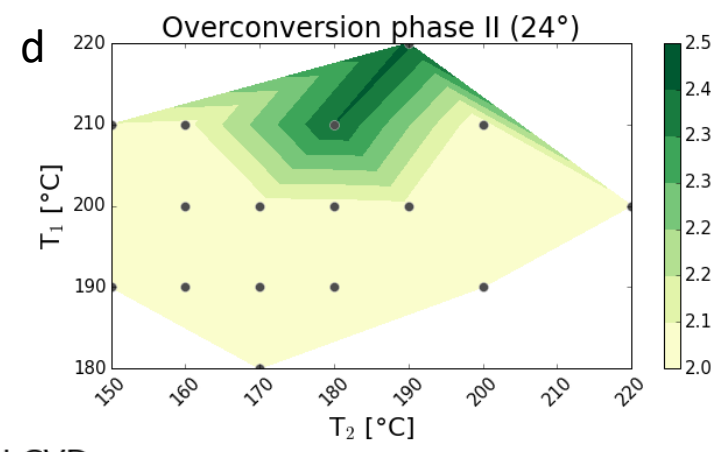

Isochronal CVD map
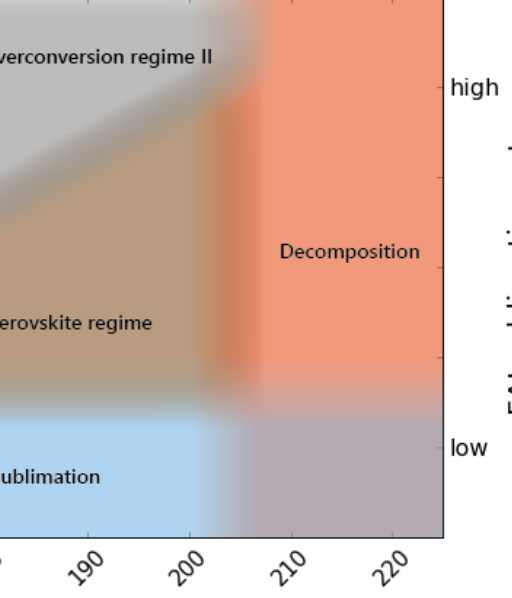

Insufficient FAI sublimation high

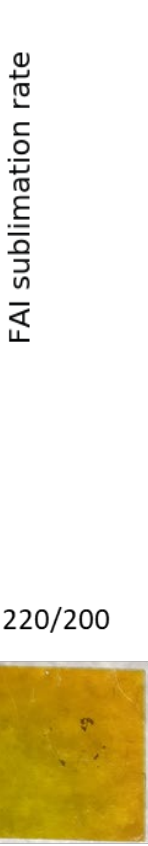

$180 / 210$

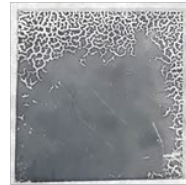

over-

conversion II full conversion over-

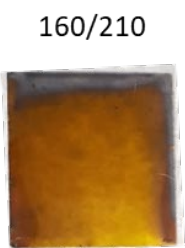

conversion I

decomposed

Figure 5: a-d) Intensity maps of selected XRD peaks after 30 minutes CVD processing time. The black dots represent the individual data points. All samples were fabricated and measured under identical conditions, varying only the temperature of the source and substrate zone in the CVD reactor. a) Perovskite (110), b) $\mathrm{Pbl}_{2}(110)$, c) overconverted phase I and d) overconverted phase II intensity map. e) Schematic isochronal CVD processing map showing five different regimes. f) Representative images of samples processed in the different regions. The apparent inhomogeneity of the sample stems from the underlying paper substrate used during photography.

\section{Device fabrication}

417

417 butyric acid methyl ester, $\mathrm{ZnO}(\mathrm{np})$ : Zinc oxide nanoparticles,

Using the novel CVD method, we fabricated a semi-transparen 418 AZO: Aluminium-doped zinc oxide)-.
perovskite solar cell with an architecture 4199 In the range of 800-1200 nm an average transmittance of 56.1\% perovskite solar cell with an architecture 420 Indium tin oxide, PTAA: Poly(triaryl amine), PCBM: Phenyl-C621 was measured (see Figure S13). The low transmittance in the near infrared region can be explained by the lack of an anti- 
a
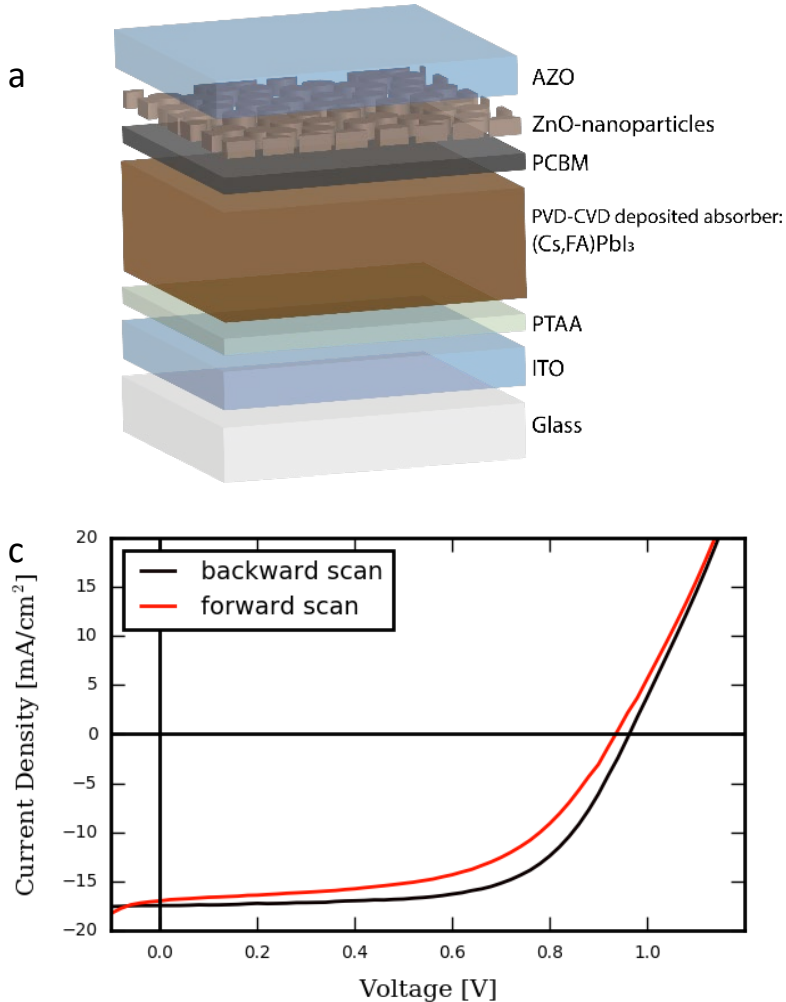

b
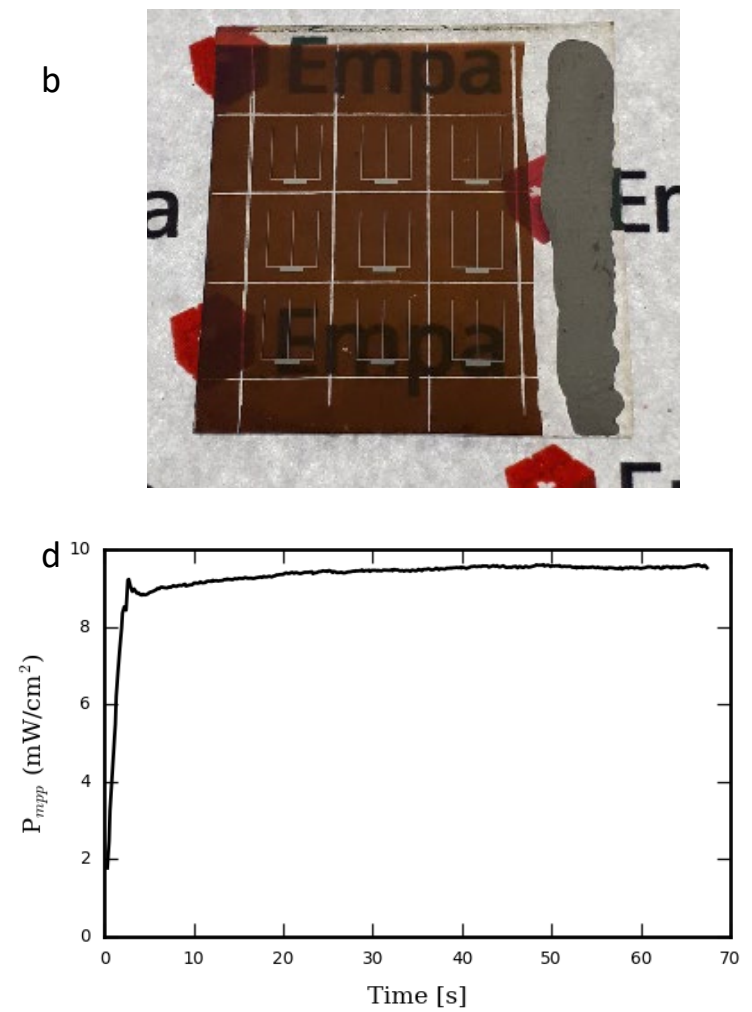

Figure 6: Semitransparent device based on a two-step sequential vapour-deposited perovskite absorber. a) Schematic architecture, b) photograph of the final device, c) J-V characteristics of forward and backward scan and d) MPP measurement of the champion device

reflection coating and the high free carrier absorption of the $\mathbf{4 5 0}$ substrate present (see Figure S14). This issue could be allevia\$d by the use of high-mobility hydrogenated indium oxide, w $|4| 6 \mid 2$ shows excellent NIR transmittance ${ }^{26,27}$.

The cell area of $0.27 \mathrm{~cm}^{2}$ was defined by mechanical scribin 4.54 schematic of the architecture and a photograph of the devit55 are shown in Figure $\mathbf{6 a}$ and $\mathbf{b}$, respectively. For the conver 456 of the precursor, the condition A (160-190) and a conver 457 time of 20 minutes was chosen in order to reach a degree 458 conversion close to $100 \%$. The measured JV characteristic 459 forward and backward direction and the maximum power p\&i60 (MPP) measurement are shown in Figure $6 \mathrm{c}$ and $\mathbf{d}$, respectiv $\$ \mathbf{6} 1$ For our champion device a power conversion efficiency of 10462 was measured when scanning in backward direction. To best of our knowledge, this is the highest reported value for the semi-transparent perovskite solar cell fabricated by the sequential two-step vapour deposition method. The stabili464t efficiency measured with maximum power point (MPP) tracki6g is $9.6 \%$. We attribute this lower performance to the significant hysteresis revealed by the forward scan (Figure 6a). statistics of the J-V parameters obtained from the backward scans of all 9 devices on the substrate are shown in Figure 15 As the device stack has not been optimized we expect that 470 device performance can be considerably increased.

We developed an isobaric-isothermal CVD process that alloyed5 us to study the reaction kinetics and the phase evolution duripo

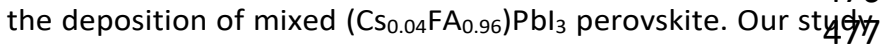

shows how the temperature of source and substrate affect the conversion reaction kinetics. We demonstrated the uniform conversion on $5 \times 5 \mathrm{~cm}$ substrates and achieved perovskite films with a growth rate of $60 \mathrm{~nm} / \mathrm{min}$, which is an important parameter from an industrial manufacturing point of view. We also show that undesired side reactions can occur during the CVD conversion that result in the formation of unfavourable phases. This imposes additional boundaries to the process as to achieve high quality perovskite layers, these reactions need to be avoided by controlling process parameters.

Using our new sequential vapour deposition method we fabricated semi-transparent PSCs with a champion PCE of $9.6 \%$ on a designated area of $0.27 \mathrm{~cm}^{2}$.

\section{Materials \& Method}

\section{Perovskite layer growth}

All chemicals were used as received, without any further purification or treatment.

To exclude substrate effects, the perovskite layers were always grown on the same substrate, namely PTAA coated ITO. The ITO substrates $\left(R_{s}<10 \Omega / \square\right.$,Zhuhai Kaivo Optoelectronics Co.) were washed by hand first, followed by ultra-sonication at $85^{\circ} \mathrm{C}$ in soap water, acetic acid solution, de-salted water and finally de-ionized water for 15 minutes each in the order given here. The ITO substrates were then dried with compressed nitrogen. A PTAA layer was prepared by spin-coating a solution of $5 \mathrm{mg}$ PTAA (Ossila) in $1 \mathrm{ml}$ of Toluene. $50 \mu \mathrm{l}$ of the solution was dropped onto the $2.5 \times 2.5 \mathrm{~cm}^{2}$ ITO substrate spinning at 6000 
$\mathrm{rpm}$ and the rotation was continued for another 30 secor $58 \mathrm{~B} 4$ The layer was dried on a hot plate at $80^{\circ} \mathrm{C}$ for 5 minutes. $\quad 535$ The ITO/PTAA substrate was then transferred to a ther巨ె36 evaporation system where $\mathrm{Pbl}_{2}$ (99.999\%, Sigma Aldrich) $58 \mathrm{~B} \mathbf{7}$ $\mathrm{CsBr}$ (99.999\%, Sigma Aldrich) were co-evaporated at 5338 evaporation pressure of $3-6 * 10 e-08$ mbar to form the mbex halide precursor layer. The deposition rate of lead iodide $5 \mathrm{~A} \otimes$ cesium bromide were kept constant at $1.7 \AA \mathrm{s}^{-1}$ and $0.07 \AA 541$ respectively. The targeted deposition thickness of the IБAd iodide was set to $250 \mathrm{~nm}$, which corresponds to a thicknes $54 B$ $10 \mathrm{~nm}$ of the cesium bromide.

544 The precursor layers were then transferred to the tubular 5.45 reactor.

For the CVD conversion, $0.2 \mathrm{~g}$ of formamidinium iodide (5A47 $>99 \%$, Greatcell Solar Materials) were loaded into a grap Бit\&8 crucible (Umicore). It was taken care that the FAl powder 549 evenly spread on the bottom of the crucible to achiev 550 constant free surface for sublimation during each run. 5 h9 crucible and the precursor coated substrate were placed at 542 same position in their individual heating zone for each run. Aftes loading, the system was pumped down until a pressure of 554 mbar was reached. Then nitrogen (99.999\%, PanGas) carrier 555 was injected via a mass flow controller such that a reverse 5056 was established (i.e., valve A closed, valve B and C open, 559 Figure 1a). The volume flow rate was set to 0.5 mbark 5 b (correspoding to approximately $30 \mathrm{sscm}$ ) which resulted in 559 equilibrium pressure of 7-8 mbar in the system during 560 following process step. With the carrier gas flowing, beth1 substrate and source heating zones were heated up to theis target temperatures ( $T_{\text {substrate }}: 150-220^{\circ} \mathrm{C}, \mathrm{T}_{\text {source }}$ : $180-22 \mathrm{G}^{\circ} \mathrm{EB}$ with high heating rate at the beginning and low heating 1554 when getting close to the target temperature to aveists overshooting. Once the target temperature were reached, 568 reverse flow was maintained for 10 minutes to estabtjis thermal equilibrium. After equilibration, the conversijis reaction was started by inverting the flow direction of 569 carrier gas to establish downstream flow (i.e., valve $A$ openend and subsequently valves $B$ and $C$ were closed). After the desired reaction time had passed, the conversion halted by once again inverting the carrier gas flow and $5 \% 3$ establishing the reverse flow. The system was kept unglen reverse flow for two minutes before the reactor was vented with nitrogen, opened and the sample was transferred t 97 क glove box to cool down and keep exposure to humid air as fow as possible.

\section{Solar cell fabrication}

580

The solar cell was fabricated with a layer structure 581 ITO/PTAA/Perovskite/PCBM/ZnO-nanoparticles/AZO/Ni-Al- 582 grid. The initial ITO/PTAA/Perovskite layer stack was deposited as described above. For the CVD conversion of the metal hafide precursor, the substrate zone was set to $160^{\circ} \mathrm{C}$ and the source zone was set to $190^{\circ} \mathrm{C}$ and the conversion reaction was allow 58. to take place for 20 minutes. The electron-transporting laye 57 was deposited on top of the perovskite by spin-coating a 588 $\mathrm{mg} / \mathrm{ml}$ solution of PCBM (PC61BM, 99.5\%, SolenneBV). 50 당 the solution were dropped at a substrate spinning speed of $4000 \mathrm{rpm}$ and rotation was continued for 30 seconds. The layer was dried for 5 minutes at $80^{\circ} \mathrm{C}$. A buffer layer of $\mathrm{ZnO}$ nanoparticles was then deposited by spin-coating. $50 \mu \mathrm{l}$ of a dispersion of $2.5 \mathrm{w} \% \mathrm{ZnO}$ nanoparticles (particle size: $12 \mathrm{~nm}$, Avantama) in isopropanol was dropped at a substrate spinning speed of 5000rpm and rotation was continued for 30 seconds. The layer was dried for 2 minutes at $80^{\circ} \mathrm{C}$. Both spin-coating and drying steps were carried out in a nitrogen filled glove box. A layer of aluminium doped zinc oxide (AZO) was deposited by RFsputtering as top contact. Subsequently $50 \mathrm{~nm}$ of nickel followed by $4 \mu \mathrm{m}$ of aluminium were e-beam evaporated through a shadow mask to form the contacting grids. The device was finalized by defining the designated area of $0.27 \mathrm{~cm}^{2}$ by mechanical scribing down to the ITO back contact.

\section{Characterization}

XRD: X-Ray diffraction patterns were measured on an X'Pert Pro in Bragg-Brentano geometry using $\mathrm{Cu}-\mathrm{K} \alpha 1$ radiation $(\lambda=1.5406$ $\AA)$, scanning from 5 to $80^{\circ}(2 \theta)$ with a step interval of $0.0167^{\circ}$. For the intensity maps the contours were extrapolated from triangulation between the individual data points.

UV-Vis: Reflectance and transmittance measurements were performed using a Shimadzu UV-Vis 3600 spectrophotometer equipped with an integrating sphere. The reflectance data was corrected for the instrumental response stemming from diffuse and specular reflections both on the sample as well as the reflectance measurements were carried out in a wavelength range from 300 to $1500 \mathrm{~nm}$.

SEM: The SEM micrographs were taken with a Hitachi S-4800 Scanning Electron Microscope and a FEI NanoSEM 230 using 5$10 \mathrm{kV}$ acceleration voltage. A thin layer $(\approx 1 \mathrm{~nm})$ of $\mathrm{Pt}$ was coated on top of the samples to avoid charging effects.

Solar cell characterization: The current density-voltage characteristics of the perovskite solar cell was measured in 4terminal sensing with a Keithley 2400 source meter. The illumination was provided with a ABA class solar simulator and was calibrated to $1000 \mathrm{~W} \mathrm{~m}^{-2}$ using a certified single crystalline silicon solar cell. The sample stage temperature was kept at 22 ${ }^{\circ} \mathrm{C}$ during the measurements. The $\mathrm{J}-\mathrm{V}$ measurements were performed in both forward (form -0.1 to $1.5 \mathrm{~V}$ ) and backward (from 1.5 to $-0.1 \mathrm{~V}$ ) direction. The external quantum efficiency of the cells was measured with a lock-in amplifier. The probing beam was generated by a chopped white source (900 W, halogen lamp, $260 \mathrm{~Hz}$ ) and a dual grating monochromator. The beam size was adjusted to ensure that the illumination area was fully inside the cell area. The shading effect of metallic grid was taken into account by including middle grid line into the illuminated area. A certified single crystalline silicon solar cell was used as the reference cell. White light bias of approximately 0.1 suns was applied during the measurement with a halogen bias lamp.

TRPL: Time-resolved photoluminescence was measured using the MicroTime 100 system from PicoQuant. A $639 \mathrm{~nm}$ pulsed laser diode (pulse width $\sim 100 \mathrm{ps}$ ) was used. The excitation density was $2 \cdot 10^{12}$ photons $\cdot \mathrm{cm}^{-2}$ pulse $\mathrm{e}^{-1}\left(\sim 3.1 \mathrm{~J} \cdot \mathrm{s}^{-1} \cdot \mathrm{cm}^{-2}\right)$. The 
590 spot size was measured by a NanoScan2 beam profiler resul 641 591 in $\sim 130 \mu \mathrm{m}$ diameter. Acquisition times were 60 seconds $84 \mathrm{~A} 2$ 592 the pulse frequency $3 \mathrm{MHz}$. For the fitting procedure a $64 \mathrm{~b}$ 593 exponential decay was used and the first $35 \mathrm{~ns}$ after the sign 64

\section{Conflicts of interest}

\section{Notes and references}

6181 S. F. Hoefler, G. Trimmel and T. Rath, Monatshefte Für Chem 679

peak were neglected.

spectroscopy was performed using 34 system from Physical Electronics with 648 below $8 \cdot 10^{-9}$ mbar. Survey spectra were recorded with6\$0 energy step size of $0.4 \mathrm{eV}$ and a pass energy of $93.9065 / 1$ Composition of the samples was analyzed using the Multi65 2 software.

Financial funding from the Innovation and Networks Executgeg Agency of the European Commission (INEA, Protero ERCISTAND, grant No. 850937), the Swiss Federal Office66 Energy (SFOE, Project CIGSPSC, grant No. SI/501805-01) and $\$ 62$ Swiss National Science Foundation (SNF, Project Bridge Pow 9643 grant No. 176552) is gratefully acknowledged.

664 discussions and suggestions regarding the building of the optical model respectively regarding the photoluminescenfo measurements. Chem. Mon., 2017, 148, 795-826.

2 M. L. Petrus, J. Schlipf, C. Li, T. P. Gujar, N. Giesbrecht, P. Mül68-1 Buschbaum, M. Thelakkat, T. Bein, S. Hüttner and P. Docamp682 Adv. Energy Mater., 2017, 7, 1700264-n/a.

3 M. A. Green, E. D. Dunlop, J. Hohl-Ebinger, M. Yoshita, N. 684 Kopidakis and A. W. Y. Ho-Baillie, Prog. Photovolt. Res. Appl.,685 2020, 28, 3-15.

4 National Renewable Energy Laboratory, NREL Best Research-6817 Efficiency Chart, https://www.nrel.gov/pv/assets/pdfs/bestresearch-cell-efficiencies.20200406.pdf, (accessed April 14, 2020).

5 P. Luo, S. Zhou, W. Xia, J. Cheng, C. Xu and Y. Lu, Adv. Mater. Interfaces, 2018, 4, 1600970.

6 R. Swartwout, M. T. Hoerantner and V. Bulović, ENERGY Environ. Mater., 2019, 2, 119-145.

7 Y. Peng, G. Jing and T. Cui, J. Mater. Chem. A, 2015, 3, 1243612442.

8 M.-H. Li, H.-H. Yeh, Y.-H. Chiang, U.-S. Jeng, C.-J. Su, H.-W. Shiu, Y.-J. Hsu, N. Kosugi, T. Ohigashi, Y.-A. Chen, P.-S. Shen, P. Chen and T.-F. Guo, Adv. Mater., 2018, 30, 1801401.

9 P. Luo, Z. Liu, W. Xia, C. Yuan, J. Cheng and Y. Lu, J. Mater. Chem. A, 2015, 3, 12443-12451.
10 X. Wei, Y. Peng, G. Jing and T. Cui, Jpn. J. Appl. Phys., 2018, 57, 052301.

11 J. Yin, H. Qu, J. Cao, H. Tai, J. Li and N. Zheng, J. Mater. Chem. A, 2016, 4, 13203-13210.

12 Y. Jiang, M. R. Leyden, L. Qiu, S. Wang, L. K. Ono, Z. Wu, E. J. Juarez-Perez and Y. Qi, Adv. Funct. Mater., 1703835-n/a.

13 G. Tong, H. Li, G. Li, T. Zhang, C. Li, L. Yu, J. Xu, Y. Jiang, Y. Shi and K. Chen, Nano Energy, 2018, 48, 536-542.

14 L. Qiu, S. He, Y. Jiang, D.-Y. Son, L. K. Ono, Z. Liu, T. Kim, T. Bouloumis, S. Kazaoui and Y. Qi, J. Mater. Chem. A, 2019, 7, 6920-6929.

15 C. Yi, J. Luo, S. Meloni, A. Boziki, N. Ashari-Astani, C. Grätzel, S. M. Zakeeruddin, U. Röthlisberger and M. Grätzel, Energy Environ. Sci., 2016, 9, 656-662.

16 G. Liu, H. Zheng, X. Xu, L.-Z. Zhu, X. Zhang and X. Pan, Chem. Mater., 2018, 30, 7691-7698.

17 M. Saliba, T. Matsui, J.-Y. Seo, K. Domanski, J.-P. Correa-Baena, M. Khaja Nazeeruddin, S. M. Zakeeruddin, W. Tress, A. Abate, A. Hagfeldt and M. Grätzel, Energy Environ. Sci., 2016, 9, 19891997.

18 A. Ummadisingu and M. Grätzel, Sci. Adv., 2018, 4, e1701402.

19 D. F. Swinehart, J. Chem. Educ., 1962, 39, 333.

20 M. R. Leyden, M. V. Lee, S. R. Raga and Y. Qi, J. Mater. Chem. A, 2015, 3, 16097-16103.

21 M. T. Hoerantner, E. L. Wassweiler, H. Zhang, A. Panda, M. Nasilowski, A. Osherov, R. Swartwout, A. E. Driscoll, N. S. Moody, M. G. Bawendi, K. F. Jensen and V. Bulović, ACS Appl. Mater. Interfaces, 2019, 11, 32928-32936.

22 V. S. Chirvony, K. S. Sekerbayev, D. Pérez-del-Rey, J. P. MartínezPastor, F. Palazon, P. P. Boix, T. I. Taurbayev, M. Sessolo and H. J. Bolink, J. Phys. Chem. Lett., 2019, 10, 5167-5172.

23 J. Li, H. Wang, X. Y. Chin, H. A. Dewi, K. Vergeer, T. W. Goh, J. W. M. Lim, J. H. Lew, K. P. Loh, C. Soci, T. C. Sum, H. J. Bolink, N. Mathews, S. Mhaisalkar and A. Bruno, Joule, DOI:10.1016/j.joule.2020.03.005.

24 T. Kirchartz, J. A. Márquez, M. Stolterfoht and T. Unold, Adv. Energy Mater., n/a, 1904134.

25 M. Stolterfoht, P. Caprioglio, C. M. Wolff, J. A. Márquez, J. Nordmann, S. Zhang, D. Rothhardt, U. Hörmann, A. Redinger, L. Kegelmann, S. Albrecht, T. Kirchartz, M. Saliba, T. Unold and D. Neher, ArXiv181001333 Phys.

26 F. Fu, T. Feurer, T. P. Weiss, S. Pisoni, E. Avancini, C. Andres, S. Buecheler and A. N. Tiwari, Nat. Energy, 2017, 2, 16190.

27 Y. Jiang, T. Feurer, R. Carron, G. T. Sevilla, T. Moser, S. Pisoni, R. Erni, M. D. Rossell, M. Ochoa, R. Hertwig, A. N. Tiwari and F. Fu, ACS Nano, 2020, 14, 7502-7512. 